\title{
Obscured clusters. I. GLIMPSE 30 - A young Milky Way star cluster hosting Wolf-Rayet stars ${ }^{\star}$
}

\author{
R. Kurtev ${ }^{1, \star \star}$, J. Borissova ${ }^{1}$, L. Georgiev ${ }^{2}$, S. Ortolani ${ }^{3}$, and V. D. Ivanov ${ }^{4}$ \\ 1 Departamento de Fisíca y Astronomía, Facultad de Ciencias, Universidad de Valparaíso, Av. Gran Bretaña 644, \\ Playa Ancha, Casilla 5030, Valparaíso, Chile \\ e-mail: radostin.kurtev@uv.cl; jura.borissova@uv.cl \\ 2 Instituto de Astronomia, Universidad Nacional Autónoma de México, Apartado Postal 70-254, CD Universitaria, \\ CP 04510, Mexico DF, Mexico \\ e-mail: georgiev@astroscu.unam.mx \\ 3 Universitá di Padova, Dipartimento di Astronomia, Vicolo dell’'Osservatorio 5, 35122 Padova, Italy \\ e-mail: sergio.ortolani@unipd.it \\ ${ }^{4}$ European Southern Observatory, Ave. Alonso de Cordova 3107, Casilla 19, Santiago 19001, Chile \\ e-mail: vivanov@eso.org
}

Received 7 November 2006 / Accepted 17 August 2007

\begin{abstract}
Context. Young massive clusters are usually deeply embedded in dust and gas. They represent excellent astrophysical laboratories for the study of massive stars. Clusters with Wolf-Rayet (WR) stars are of special importance, since this enables us to study a coeval WR population at a uniform metallicity and known age.

Aims. We started a long-term project to search the inner Milky Way for hidden star clusters and to study them in detail. GLIMPSE 30 (G30) is one of these clusters. It is situated near the Galactic plane $\left(l=298^{\circ} .756, b=-0.408\right)$ and we determine its physical parameters and investigate its high-mass stellar content especially WR stars.

Methods. Our analysis is based on SOFI/NTT $J_{\mathrm{S}} H K_{\mathrm{S}}$ imaging and low resolution $(R \sim 2000)$ spectroscopy of the brightest cluster members in the $\mathrm{K}$ atmospheric window. For the age determination we applied isochrone fits for MS and Pre-MS stars. We derived stellar parameters of the WR stars candidates using a full nonLTE modeling of the observed spectra.

Results. Using a variety of techniques we found that G30 is very young cluster, with age $t \approx 4$ Myr. The cluster is located in the Carina spiral arm, it is deeply embedded in dust and suffers reddening of $A_{V} \sim 10.5 \pm 1.1 \mathrm{mag}$. The distance to the object is $d=7.2 \pm 0.9 \mathrm{kpc}$. The mass of the cluster members down to $2.35 \mathcal{M}_{\odot}$ is $\sim 1600 \mathcal{M}_{\odot}$. The cluster's MF for the mass range of 5.6 to $31.6 \mathcal{M}_{\odot}$ shows a slope of $\Gamma=-1.01 \pm 0.03$. The total mass of the cluster obtained by this MF down to $1 \mathcal{M}_{\odot}$ is about $3 \times 10^{3} \mathcal{M}_{\odot}$. The spectral analysis and the models allow us to conclude that at least one Ofpe/WN and two WR stars can be found in G30. The WR stars are of the WN6-7 hydrogen rich type with progenitor masses of more than $60 \mathcal{M}_{\odot}$.

Conclusions. G30 is a new member of the family of young Galactic clusters hosting WR stars. It is a factor of two to three less massive than some of the youngest super-massive star clusters like Arches, Quintuplet and the Central cluster and is their smaller analog.
\end{abstract}

Key words. Galaxy: open clusters and associations: general - stars: Wolf-Rayet

\section{Introduction}

Stars rarely form in isolation. It is well known that most of the stars in our Galaxy, and in nearby galaxies, ever born in groups ranging from small associations and open clusters, compact young massive clusters to old globulars. Young clusters are often difficult to find because they can be heavily embedded in dust, making them visible only in the infrared. We embarked on a long-term project to search the inner Milky Way for hidden star clusters and to study them in details (Borissova et al. 2003, 2005, 2006; Ivanov et al. 2002, 2005). This project was based on the 2MASS mission (Skrutskie et al. 2006), taking advantage of the reduced extinction in the near-IR. Recent advances in

^ Based on observations collected with the New Technology Telescope of the ESO within observing program 77.D-0089.

$\star \star$ "Centro de Astrofísica de Valparaśo". Visiting astronomer ESO La Silla Paranal Observatory.
mid-IR instrumentation have made it possible to carry out allsky IR surveys in this spectral region too. The recent Spitzer Space Telescope Galactic Legacy Infrared Mid-Plane Survey Extraordinaire (GLIMPSE, Benjamin et al. 2003) offers an excellent opportunity to carry out a deep census of such objects. GLIMPSE is an excellent tool for finding obscured clusters in the Galactic disk because the extinction in the mid-IR is a factor of 2-5 lower than in the near-IR. Recently, Mercer et al. (2005) carried a comprehensive search for clusters in the mid-IR. They used the point source catalog of GLIMPSE and reported 92 cluster candidates. In our project we continued the investigation of some of these objects using near-IR imaging and low resolution IR spectroscopy.

Massive stars themselves play an important role in the ecology of galaxies, providing a major source of ionizing UV radiation, mechanical energy and chemical enrichment. Wolf-Rayet (WR) stars represent an evolved phase of the most massive 


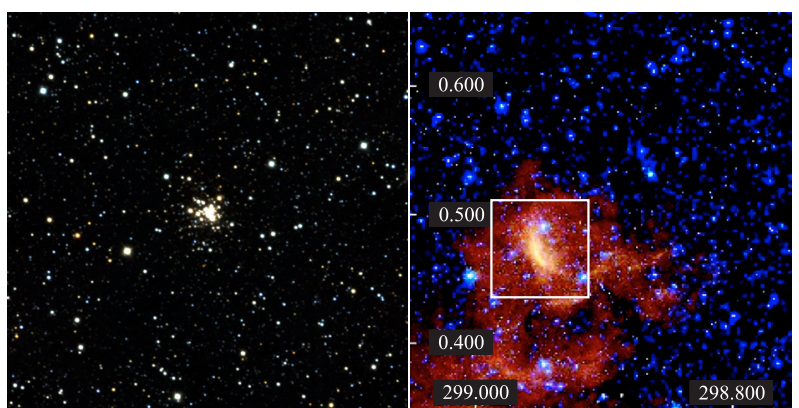

Fig. 1. Pseudo-true color images of the G30. The left panel image is composed from $J_{\mathrm{S}}$ - blue, $H$ - green and $K_{\mathrm{S}}-$ red SofI/NTT images covering $4.92 \times 4.92$ centered at the cluster. The right panel is composed from $3.6 \mu \mathrm{m}$ - blue, $5.8 \mu \mathrm{m}$ - green and $8.0 \mu \mathrm{m}$ - red Spitzer Space Telescope images. The SofI field of view from the left panel is marked with a square. The Galactic coordinates $l$ and $b$ are shown on the right panel.

stellar population, and are characterized by high mass loss rates from fast and dense winds. Their short lifetimes and high luminosities make them excellent tracers of active recent star formation. However, serious gaps in our understanding of massive stars exist because of their rapid evolution and rarity. WRs in clusters are particularly interesting because this enables us to study a coeval population with uniform metallicity. There are about three hundred known WR stars in our Galaxy (van der Hucht 2006). At the same time from thousands of known open clusters and stellar associations only small number contain WRs and only a few of them host more than one such star. This is because a typical cluster $\left(\sim 10^{3} \mathcal{M}_{\odot}\right)$ contains a limited number of stars more massive than $\sim 30-35 \mathcal{M}_{\odot}$ that can evolve to the WR phase. Therefore, most cluster WRs are concentrated in the high-mass clusters, i.e. Arches, Quintuplet, Westerlund 1.

Here we report our first results for GLIMPSE 30 (hereafter G30; Fig. 1) - a compact young cluster located near the Galactic plane $\left(l=298^{\circ} .756, b=-0^{\circ} .408\right)$ containing many massive stars including at least one Of and three Wolf-Rayet members. The presence of at least three WRs in G30 puts this cluster into the family of WR rich clusters. The cluster membership provides excellent observational constraints upon the ages and initial masses of this type of stars. In this paper we present the main results of our photometric and spectral analysis of the cluster and the newly discovered WRs.

\section{Observations and data reduction}

All observations were obtained with SofI/NTT (Son of ISAAC; Moorwood et al. 1998). The instrument is equipped with a Hawaii $\mathrm{HgCdTe} 1024 \times 1024$ detector, with a pixel scale of $0.288 \operatorname{arcsec} \mathrm{px}^{-1}$. For the spectroscopy we used a 1 arcsec slit and the medium-resolution grism. The seeing for all observations was $1-1.5$ arcsec and the sky was photometric.

Deep $J_{\mathrm{S}} H K_{\mathrm{S}}$ imaging of G30 were carried out on April 15, 2006. We took 16 images in each filter in jittering mode with 3 arcmin jitter box size to ensure that there is no overlapping of the cluster on different images. Each individual image was the average of $3 \times 20 \mathrm{~s}$ frames in $J_{\mathrm{S}}, 6 \times 10 \mathrm{~s}$ frames in $H$, and $10 \times 6 \mathrm{~s}$ frames in $K_{\mathrm{S}}$. The total integration time was $16 \mathrm{~min}$ in each filter. To obtain photometry of the brightest cluster members we took additional shallow images on August 10, 2006, using the same jittering pattern but shorter integrations: $5 \times 1.182 \mathrm{~s}$. The total integration time was $1.58 \mathrm{~min}$ in each filter. The data reduction included: sky-subtraction, flat-fielding, image alignment and combination into a single final image for each filter. A 3 -color composite image of G30 is shown in Fig. 1.

The stellar photometry of the final images was carried out with ALLSTAR in DAOPHOT II (Stetson 1987). The typical photometric errors vary from 0.01 mag for stars with $K_{\mathrm{S}} \sim 10 \mathrm{mag}$ to $0.10 \mathrm{mag}$ for $K_{\mathrm{S}} \sim 18 \mathrm{mag}$ and $0.15 \mathrm{mag}$ for $K_{\mathrm{S}} \sim 19 \mathrm{mag}$. The photometric calibration was performed by comparing our instrumental magnitudes with the 2 MASS measurements of about 1330 stars, covering the color range $0.0 \leq J_{\mathrm{S}}-K_{\mathrm{S}} \leq 3.0 \mathrm{mag}$ and magnitude range $10 \leq K_{\mathrm{S}} \leq 14.5 \mathrm{mag}$. A least squares fit of the instrumental $j h k$ magnitudes to the standard 2MASS system gave the following relations:

$$
\begin{aligned}
& \left(J_{\mathrm{S}}-K_{\mathrm{S}}\right)-(j-k)=-1.302_{( \pm 0.005)} \\
& J_{\mathrm{S}}-j=0.005_{( \pm 0.007)} \times\left(J_{\mathrm{S}}-K_{\mathrm{S}}\right)-2.435_{( \pm 0.003)} \\
& H-h=-0.021_{( \pm 0.008)} \times\left(J_{\mathrm{S}}-K_{\mathrm{S}}\right)-2.457_{( \pm 0.003)} \\
& K_{\mathrm{S}}-k=-0.004_{( \pm 0.006)} \times\left(J_{\mathrm{S}}-K_{\mathrm{S}}\right)-1.134_{( \pm 0.005)}
\end{aligned}
$$

The final photometry list contains equatorial coordinates and $J_{\mathrm{S}} H K_{\mathrm{S}}$ magnitudes of 7469 stars with photometric errors less than 0.15 mag. Artificial star tests show that the $80 \%$ completeness limit of the photometry is at $J_{\mathrm{S}}=19.1$ and $K_{\mathrm{S}}=17.4$.

The spectra were obtained on Apr 14, 2006. They cover the region from $\sim 2.00$ to $\sim 2.35 \mu \mathrm{m}$. The slit was aligned on a sample of bright cluster members and the telescope was nodded along the slit between the exposures. We obtained 16 images of $150 \mathrm{~s}$ each, in one slit position. In total, spectra of 8 stars were extracted from the data. To correct for the telluric absorption we observed the star HIP59642 (HD106290) - a solar nearanalog of spectral class G1V. The reduction process included: sky subtraction, flat fielding, geometric distortion correction, image alignment and combination into a final 2-dimensional spectrum. Next, we extracted 1-dimensional spectra, wavelength calibrated them, and corrected them for the telluric absorption by multiplying by the telluric standard. After this the target spectra were multiplied by a solar spectrum to remove the artificial emission lines due to the intrinsic absorption features in the spectra of the standard (see Maiolino et al. 1996).

\section{3. $\mathrm{J}_{\mathrm{S}} \mathrm{HK}_{\mathrm{s}}$ color-magnitude and two-color diagrams}

\subsection{Field-star decontamination}

The cluster G30 is located extremely close to the Galactic plane and therefore is in a crowded stellar field. However, it is rather concentrated and occupies a small area with diameter $\sim 1.3$ arcmin, always near the center of the SofI field of view. This allows us to define and subtract reliable the fore- and background field star contamination.

Photometric and spatial criteria were used to select probable cluster members. First, only the stars in a circle with radius $R=150 \mathrm{px}(\sim 43 \operatorname{arcsec})$, similar to the apparent cluster size, and centered on the cluster were chosen. This limits the candidate members to 592 stars. The color-magnitude diagram (CMD) of this sample is shown on the left panel of Fig. 2. The locus of the cluster Main Sequence (MS) stars can be easily seen at $1.5<\left(J_{\mathrm{S}}-K_{\mathrm{S}}\right)<2 \mathrm{mag}$. Some fore- and background stars are also present in this diagram. In order to define their locus, the CMD of the stars falling into a circle with the same radius but centered 100 arcsec to the North-East from the cluster's center is shown on the right panel of Fig. 2. The contamination becomes significant only at $K>17$ mag while the rest of the cluster locus is almost entirely devoted to field stars. 


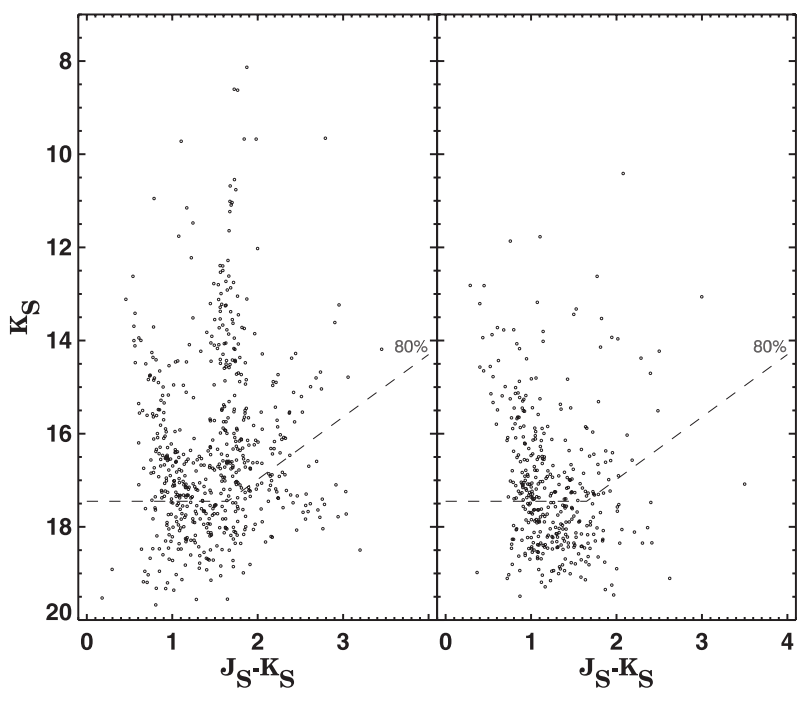

Fig. 2. $\left(J_{\mathrm{S}}-K_{\mathrm{S}}, K_{\mathrm{S}}\right)$ color-magnitude diagrams of the stars within the circle with radius $R=43.2 \operatorname{arcsec}(R=150 \mathrm{px})$ around G30 (left panel) and a comparison field (right panel). See Sect. 3.1 for details.

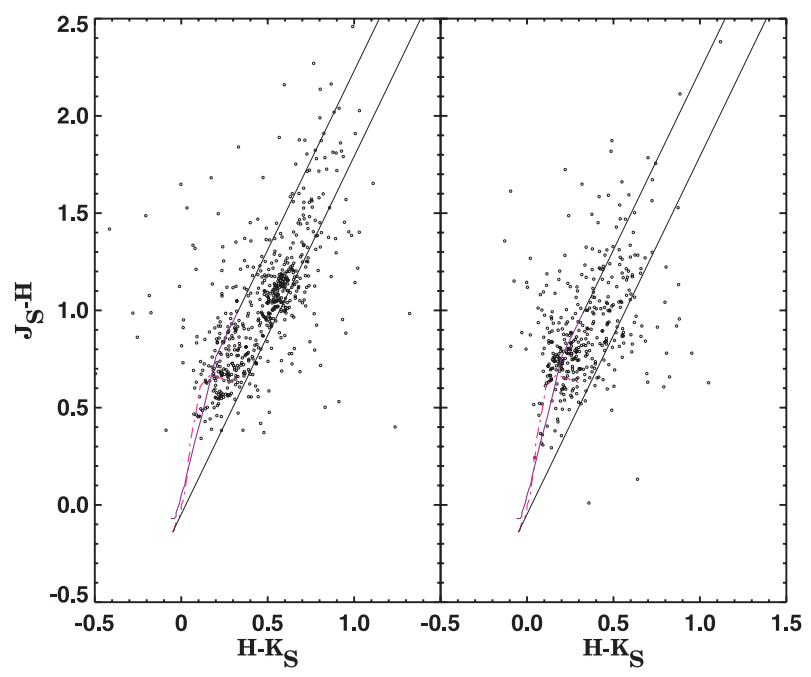

Fig. 3. $\left(H-K_{\mathrm{S}}, J_{\mathrm{S}}-H\right)$ two-color diagrams of the stars within the circle with radius $R=43.2 \operatorname{arcsec}(R=150 \mathrm{px})$ around G30 (left panel) and the comparison field (right panel). The continuous and dotted lines represent the sequence of the zero-reddening stars of luminosity classes I (Koornneef 1983) and V (Schmidt-Kaler 1982), respectively. Reddening vectors for O5V and M5I stars are also shown.

The majority of the cluster candidate members occupies a well-defined locus on the two-color diagram (TCD) as well, at $H-K_{\mathrm{S}} \sim 0.6 \mathrm{mag}$ and $J_{\mathrm{S}}-H \sim 1.1 \mathrm{mag}$. Figure 3 shows that there is only a small number of field stars falling in the cluster locus on the right panel of the same figure where the TCD of the comparison field (defined above) is shown.

The decontamination procedure was based on the color and magnitude distributions of the field stars in five stellar fields around G30, to improve the field star statistics. Each field covers the same area as the cluster. Their combined CMD was divided into bins and the number of stars in each bin was divided by five to normalize the combined field area to the cluster area. Then, we randomly subtracted from the corresponding bin of the cluster's CMD as many stars as were present in the corresponding field CMD bin. The bin sizes varied depending on the number of stars per bin. In other words, we merged nearby bins, if they
Table 1. Of and WR stars in G30.

\begin{tabular}{cccccc}
\hline \hline ID & $\alpha_{2000}$ & $\delta_{2000}$ & $J_{\mathrm{S}}-H$ & $H-K_{\mathrm{S}}$ & $K_{\mathrm{S}}$ \\
\hline 1 & $12: 14: 33.91$ & $-62: 58: 48.7$ & 1.250 & 0.732 & 9.676 \\
2 & $12: 14: 33.09$ & $-62: 58: 51.0$ & 1.194 & 0.648 & 9.674 \\
3 & $12: 14: 31.76$ & $-62: 58: 51.9$ & 1.188 & 0.577 & 8.625 \\
4 & $12: 14: 31.54$ & $-62: 58: 54.3$ & 1.152 & 0.573 & 8.605 \\
\hline
\end{tabular}

contained less than 2 stars. The total number of stars subtracted throughout the decontamination was 191.

\subsection{Analysis of the decontaminated diagrams}

The decontaminated CMD and TCD of the cluster are shown in Fig. 4. The CMD morphology presents an extended MS reaching down to $\sim 17 \mathrm{mag}$. The majority of these stars form a welldefined compact sequence on the TCD around $H-K_{\mathrm{S}} \sim 0.6$ mag. The spectroscopically confirmed Of/WN and WR stars (marked with diamonds) are among the brightest stars in the field and they are located to the right of the MS, as expected for evolved objects. There is a hint of deviation of the most massive stars from the MS at $10<K_{\mathrm{S}}<12 \mathrm{mag}$.

The CMD shows a population of 45 likely pre-main sequence stars (PMS; marked with crossed circles) at $1<K_{\mathrm{S}}<17 \mathrm{mag}$ and $2<J_{\mathrm{S}}-J_{\mathrm{S}}<2.5 \mathrm{mag}$. We cannot exclude the possibility that some of them might be unresolved binaries or MS stars subjected to higher differential reddening. To constrain the age and masses of these stars we applied isochrone fits with PMS tracks of different ages: 0.1, 1, 4, 7, and 10 Myr from Siess et al. (2000) shifted by the obtained distance modulus and reddening to the cluster (see Sect. 5 for details). Considering the photometric errors and the crowding effects we reach the tentative conclusion that the majority of the PMS candidates have ages between 1 and $10 \mathrm{Myr}$.

A non-negligible number of objects (23) fall to the right of the reddening vector for the reddest stars on the TCD. Most likely they have an excess due to circumstellar envelopes and/or discs. We marked with solid dots those stars that are more than $3 \sigma \sim 0.12$ mag redder than the reddening vector for the reddest MS stars.

\section{Wolf-Rayet stars}

The Ofpe/WNL and WR members of G30 are marked with diamonds in Fig. 4. Their coordinates and infrared broadband properties are listed in Table 1, and their spectra are plotted in Fig. 5. The $S / N$ at $\lambda=2.07 \mu \mathrm{m}$ for the stars 1 to 4 is $90,80,80$, and 60 , respectively.

The equivalent widths of the emission lines were measured from the normalized spectra. To quantify the subtypes of WR stars, we compared our data with the near-IR spectral atlases of Crowther \& Smith (1996) and Figer et al. (1997), following the spectral classification scheme of Crowther et al. (2006). The results are summarized in Table 2.

Our analysis shows that star No. 1 is WN7, based on the presence of strong N III at $2.116 \mu \mathrm{m}$. The He II line at $2.189 \mu \mathrm{m}$ is weaker than $\mathrm{Br} \gamma$, but $\mathrm{He}$ I lines are also weak.

Star No. 2 is WN6 because the observed ratio He II $2.189 \mu \mathrm{m} / \mathrm{Br} \gamma>1$ shows a lack of hydrogen. The spectrum is similar to that of WR136 = HD192163 (van der Hucht 2001).

Star No. 3 is particularly interesting. The presence of $\mathrm{Br} \gamma$ emission, the weak He I lines and the lack of He II lines in 

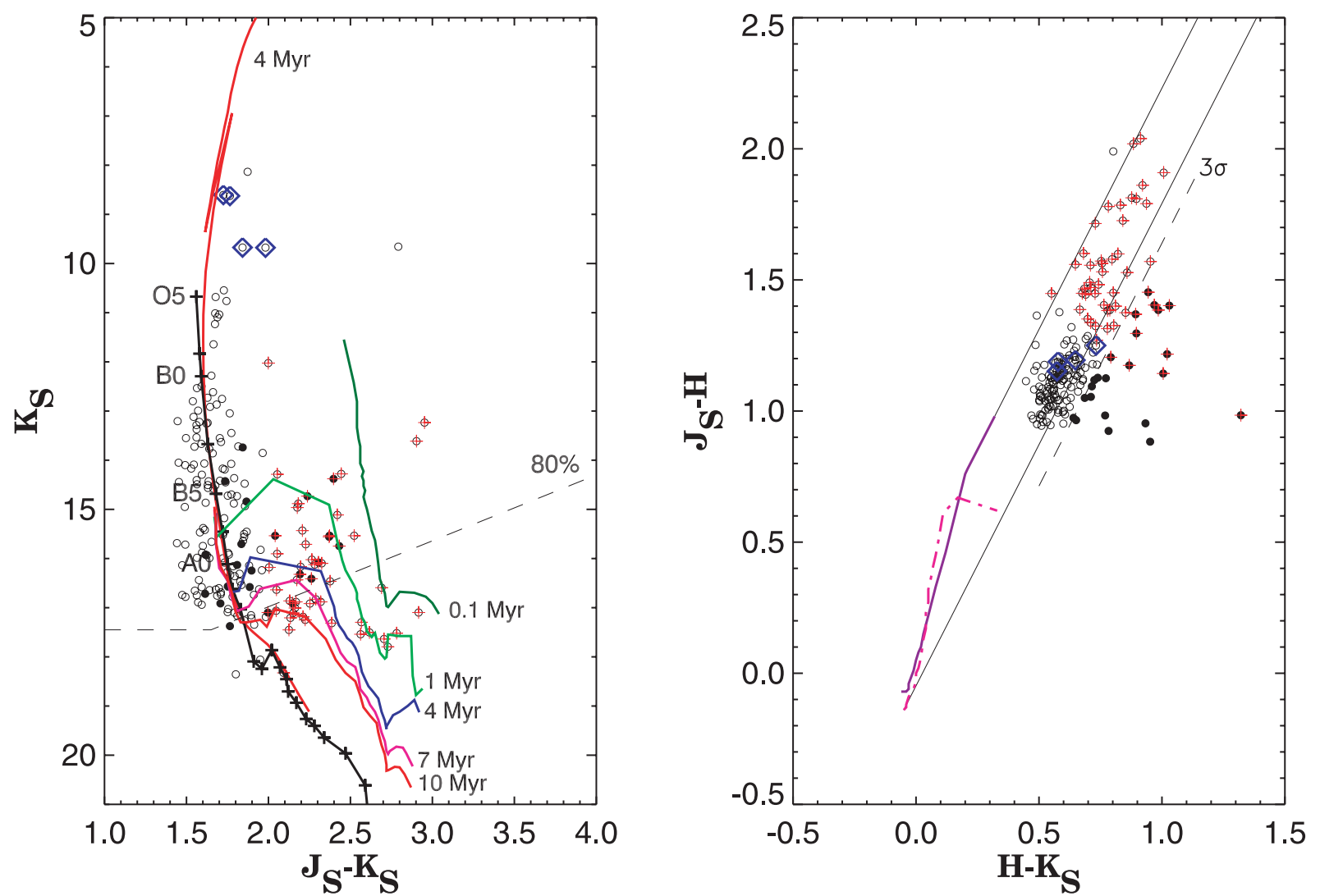

Fig. 4. Left: decontaminated $\left(J-K_{\mathrm{S}}, K_{\mathrm{S}}\right)$ color-magnitude diagram. An isochrone for $4 \mathrm{Myr}$ from Girardi et al. (2002) and PMS isochrones for various ages and solar metallicity from Siess et al. (2000) are shown. The $80 \%$ completeness limit of the photometry is marked with a dashed line. Solid dots indicate stars with color excess, as defined in the right panel. Right: decontaminated $\left(H-K_{\mathrm{S}}, J_{\mathrm{S}}-H\right)$ two-color diagram. The continuous and dotted lines represent the sequence of the zero-reddening stars of luminosity classes I (Koornneef 1983) and V (Schmidt-Kaler 1982), respectively. Reddening vectors for O5V and M5I stars are also shown. The probable PMS stars are marked with crosses. The $3 \sigma$-limit line of the reddening vector for OV stars is drawn $\approx 0.12$ mag to the right of the reddening vector. The stars redder than this line show color excess and are marked with solid circles.

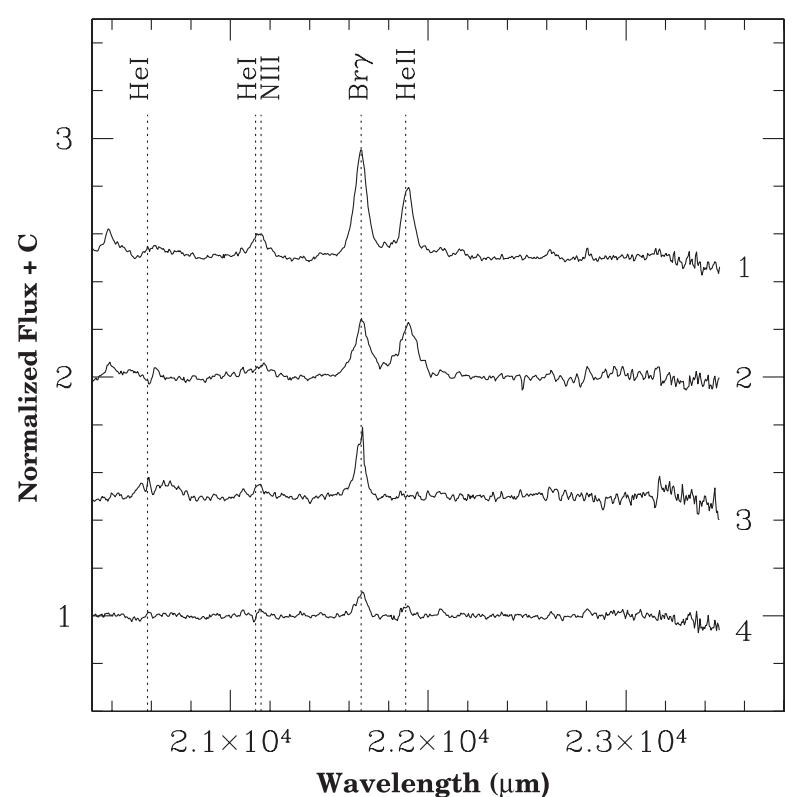

Fig. 5. Infrared spectra of the G30 stars. The spectra are continuum normalized and shifted vertically by 0.5 for display purposes. The $S / N$ at $\lambda=2.07 \mu \mathrm{m}$ for the stars 1 to 4 is approximately $90,80,80$, and 60 , respectively.

absorption and in emission suggest that it is Ofpe/WNL. The spectrum resembles those of HD 47129 (O8p), HD 148937
(O6fp), HD 152408 (O8Iafpe), and HD 269582 (Ofpe/WN9). There are only a few known stars of this type in the Galaxy. Interestingly, Ofpe stars also reside in clusters: in Quintuplet (Figer et al. 1999) and in the Galactic Center cluster (Cotera et al. 1999). This warrants further study of this star.

Star No. 4 shows a strong He II line (as strong as Br $\gamma$ ), but it also has an He I and no N III line. The measured line intensities (Table 2) suggest a tentative WN7-8 classification. Unfortunately, the star was located at the edge of the slit and its spectrum has the lowest $S / N$ ratio of all four WR and $\mathrm{O}$ star candidates. We compared this spectrum with the template spectra of WN and O stars given in Conti et al. (1995), Hanson et al. (1996), Figer et al. (1997) and Crowther et al. (2006), smoothing the templates to the resolution of our data. The closest matches are with WR131 (WN+a) and HD16691 (O4 If $\left.{ }^{+}\right)$, shown in Fig. 6. Note that the intensities of He II and $\mathrm{Br} \gamma$ are higher in the template spectra and they have much more reliable $\mathrm{He}$ I + N III lines. This comparison also shows similarities to the WN7 class but the He II emission suggest an early or mid-O star.

Another four stars fall onto the slit by chance but their $S / N$ is too low for a quantitative analysis and they show no strong emission lines.

The spectral classification described above gives a rough estimate of the temperature and luminosity of the stars. The precision of these estimates is related to the intrinsic variation within the classes. Unfortunately, the parameters of the WR stars are 
Table 2. Spectral classification of Of and WR stars in G30. For each object the equivalent widths (first line) and FWHM (second line) in $\AA$ for prominent near-IR lines in the spectrum are presented. The central wavelengths of the lines are in $\mu \mathrm{m}$.

\begin{tabular}{llcccccl}
\hline \hline Star & He I & N V & He I + N III & He II + Br $\gamma$ & He II & He II / Br $\gamma$ & Type \\
& 2.058 & 2.110 & 2.115 & 2.165 & 2.189 & & \\
\hline 1 & P Cyg & no & 8.97 & 23.34 & 14.84 & 0.67 & WN7 \\
& & & 97.2 & 68.7 & 60.7 & & weak \\
2 & PCyg & no & -2.6 & 10.56 & 15.36 & 1.45 & WN6 \\
& & & 82 & 74 & 89 & & weak \\
3 & PCyg & 0.39 & 0.98 & 6.26 & no & 0.67 & Ofp/WN \\
& & 19 & 30 & 47 & & & $?$ \\
4 & absorb. & no & no & 2.77 & 2.03 & 0.83 & WN7-8 \\
& -2.53 & & & 45 & 49 & & weak \\
\hline
\end{tabular}

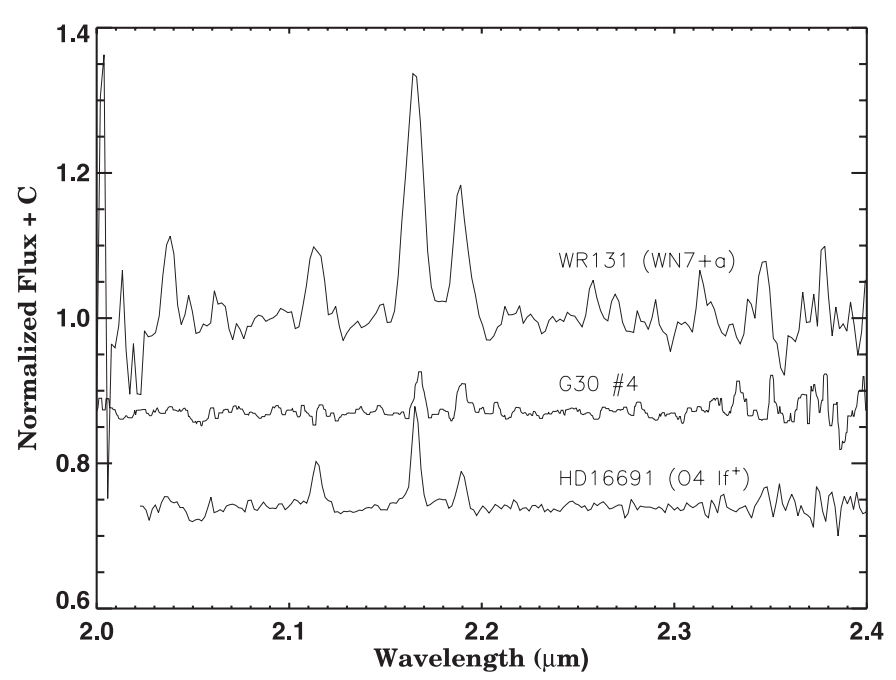

Fig. 6. Comparison of the spectrum of star \#4 with template spectra of WR131 (WN+a) and HD16691 (O4 If $\left.^{+}\right)$from Figer et al. (1997) and Hanson et al. (1996).

not truly homogeneous. Later in the text we use WR stars as distance indicators, so having better estimates is crucial.

The limited number of emission lines in the $\mathrm{K}$ atmospheric window makes the line ratios less reliable than a full line modeling. We used the CMFGEN model (Hillier \& Miller 1998) to obtain synthetic line profiles. Even though the spectra do not contain a lot of spectral features we run models with complex atoms including $\mathrm{CNO}, \mathrm{Si}, \mathrm{P}, \mathrm{S}$ and Fe because they have an effect on the electron density, temperature, etc. and therefore on the properties of the hydrogen and helium lines.

The two main lines in our spectra $\mathrm{Br} \gamma$ and $\mathrm{He}$ II $2.18 \mu \mathrm{m}$ are sensitive to both temperature and mass loss rate. Their ratio is sensitive to the helium abundance. To measure all parameters simultaneously we calculated a grid of models spanning a range of temperatures from 35000 to $40000 \mathrm{~K}$ and mass loss rates from $1 \times 10^{-6}$ to $1.5 \times 10^{-5} \dot{\mathcal{M}} / \mathrm{yr}$. The chemical composition was set to solar except for $\mathrm{He} / \mathrm{H}$ which was set to $\mathrm{He} / \mathrm{H}=1.0$, in terms of number of atoms. Initially, the luminosity of each star was set to $\log L / L_{\odot}=6.0$ and the terminal velocity to $V_{\infty}=1000 \mathrm{~km} \mathrm{~s}^{-1}$, determined from the width of the spectral lines. There are no lines with good P Cyg profiles in the K spectrum, so the value of $V_{\infty}$ should be treated with caution. We discus the values of the luminosity below.

The synthetic spectra were degraded to the resolution of the observed spectra, using the profile of the arc lamps as a

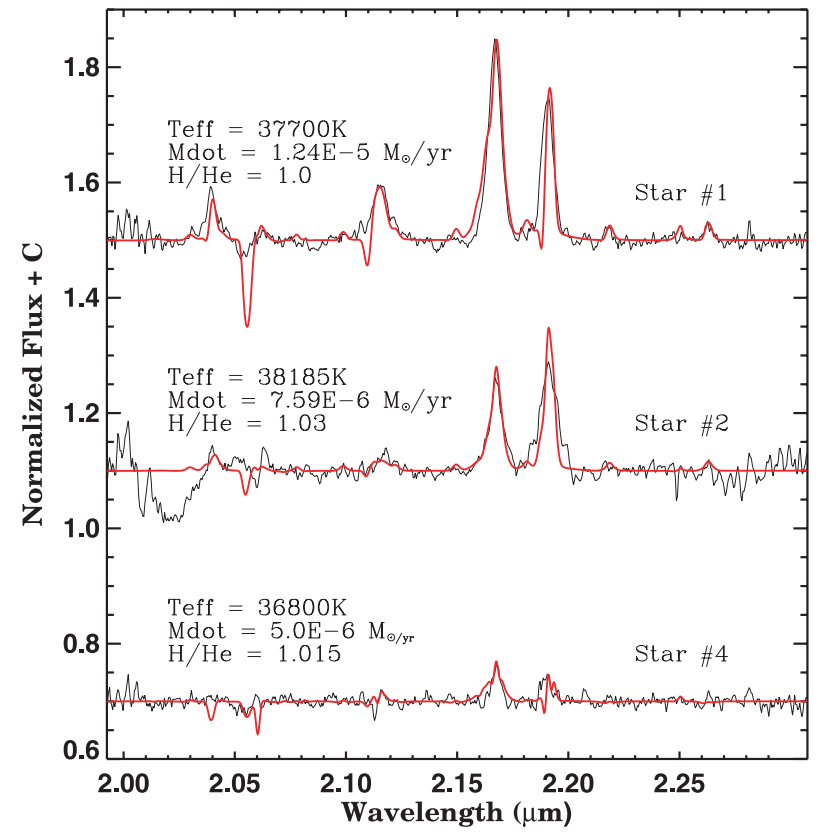

Fig. 7. CMFGEN model (Hillier \& Miller 1998) fit to the observed spectra of stars \#1, \#2, and \#4.

measure of the instrumental profile (Fig. 7). Then, we measured the intensities of $\mathrm{Br} \gamma, \operatorname{HeI} 2.05 \mu \mathrm{m}$, He I $2.11 \mu \mathrm{m}$ and He II $2.18 \mu \mathrm{m}$ on both the models and the observed spectra. The grid of models defines a surface in $T_{\text {eff }}$ vs. $\dot{\mathcal{M}}$ space. The observed value of each spectral feature is an iso-line on that surface. The crossing of the iso-lines, determined by different features, sets the parameters that reproduce simultaneously all measured features. The temperature depends mainly on the ratio of He I/He II lines while the intensity of Br $\gamma$ is mainly related to $\dot{\mathcal{M}}$. The intensity of He II $2.18 \mu \mathrm{m}$ is sensitive to $T_{\text {eff }}, \dot{\mathcal{M}}$ and to the helium composition but the first two are already constrained by the other parameters. We had to adjust the $\mathrm{He} / \mathrm{H}$ ratio to reproduce this line well.

As pointed out above, the values of the stellar luminosities were assumed. There is no easy way to determine the exact luminosity from the spectra alone. We broke the luminosityradius-mass loss rate degeneracy applying the relation between modified wind momentum $\Pi=\dot{\mathcal{M}} \mathrm{V}_{\infty} R_{*}^{0.5}$ and the luminosity (Kudritzki et al. 1995). The derived $T_{\text {eff }}, \dot{\mathcal{M}}$ and $V_{\infty}$ set a family of possible modified wind momenta and stellar luminosities, where the stellar radius is a free parameter. We calculated the 


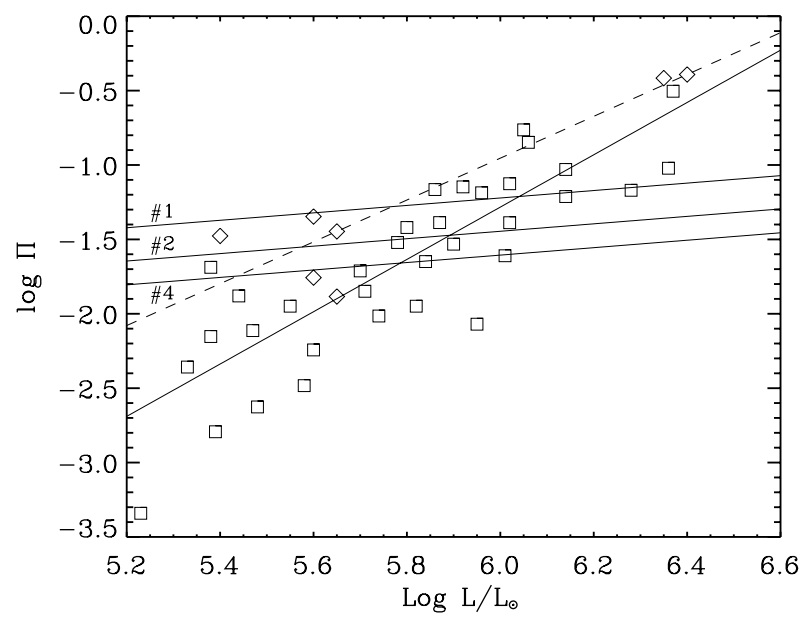

Fig. 8. Modified wind momentum $\Pi$ versus luminosity $L$ diagram for a range of radii, for stars \#1, \#2, and \#4. The crosspoint between the individual line for each star and the observed $\log L-\log \Pi$ relation determines the stellar luminosity. WNha stars from Hamann et al. (2006) are marked with diamonds. The least square fit to these data alone is shown with a dashed line. O stars from Lamers et al. (1999) are marked with squares. The least square fit to all data is shown with a solid line.

Table 3. Physical parameters of WR stars in G30. The bolometric corrections BC from Crowther et al. (2006), the absolute $K$ magnitude and the true distance modulus, obtained from WR stars are also listed.

\begin{tabular}{cccccccc}
\hline \hline Star & $\begin{array}{c}\log L \\
{\left[L_{\odot}\right]}\end{array}$ & $\begin{array}{c}T_{\text {eff }} \\
{[\mathrm{K}]}\end{array}$ & $\begin{array}{c}\log \dot{\mathcal{M}} \\
{\left[\mathcal{M}_{\odot} / \mathrm{yr}\right]}\end{array}$ & $\mathrm{H} / \mathrm{He}$ & $\begin{array}{c}\mathrm{BC} \\
{[\mathrm{mag}]}\end{array}$ & $\begin{array}{c}M_{K} \\
{[\mathrm{mag}]}\end{array}$ & $\left(K-M_{K}\right)_{0}$ \\
\hline 1 & 6.04 & 37700 & -4.91 & 1.000 & -3.8 & -6.55 & 15.065 \\
2 & 5.91 & 38185 & -5.12 & 1.030 & -4.4 & -5.62 & 14.134 \\
4 & 5.81 & 36800 & -5.30 & 1.015 & -3.6 & -6.18 & 13.625 \\
\hline
\end{tabular}

possible $\Pi$ and $L$ for a range of radii and plotted them on the Kudritzki et al. (1995) diagram together with data for WNha stars from Hamann et al. (2006). The crossing between this line and the observed $\log L-\log \Pi$ relation determines the stellar luminosity which gives the same emitted spectrum and satisfies the $\log L-\log \Pi$ relation - the least square fit to the data (open diamonds) and is shown in Fig. 8 with a dashed line. The obtained $\log L / L_{\odot}$ is $5.77,5.57$ and 5.43 for stars \#1, \#2, and \#4 respectively. However, the sample of WN stars containing hydrogen is very small and we added the data of O-type stars given in Lamers et al. (1999; shown with squares) to improve the statistics. The new relation based on the expanded dataset is shown with a continuous line in Fig. 8. The luminosities are listed in Table 3 and we will use them as our final estimates.

This technique allowed us to determine the temperature and the mass loss rate of the strong He II $2.18 \mu \mathrm{m}$ emission stars \#1, $\# 2$, and \#4. We cross-checked the model predictions with the features expected in $K$ spectra. The N III at $2.115 \mu \mathrm{m}$ is heavily blended with a He I line which makes the accurate determination of the nitrogen composition difficult. Nevertheless, the profile fitting favors an increase of $\mathrm{N}$ abundance by a factor of 10 with respect to solar. Higher resolution spectra are required to achieve better precision. The C IV $2.07 \mu \mathrm{m}$ is absent from the observed spectra, which suggests a reduction of the carbon abundance by a factor of at least 10. In general, these three stars have increased mass loss rates and relatively low terminal velocity, higher helium abundance and reduced carbon. All these characteristics make the first two of them good candidates to be hydrogen rich WN stars. The type of the third star cannot be constrained with our data.

\section{Discussion}

\subsection{Reddening and distance}

We used photometric and positional criteria to select probable cluster members. The candidates occupy a well-defined locus on the color-color diagram at $H-K_{\mathrm{S}} \sim 0.5-0.6 \mathrm{mag}$ and $J_{\mathrm{S}}-H \sim 0.9-1.2 \mathrm{mag}$ allowing us to determine the reddening to the cluster. First, we simply measured the color excesses of this locus on the color-color diagram with respect to the sequences of unreddened MS stars (Schmidt-Kaler 1982), obtaining $E(H-$ $\left.K_{\mathrm{S}}\right)=0.60 \pm 0.06 \mathrm{mag}$ and $E\left(J_{\mathrm{S}}-H\right)=1.10 \pm 0.06 \mathrm{mag}$, corresponding to $A_{K_{\mathrm{S}}}=1.12 \pm 0.08 \mathrm{mag}$ and $A_{V}=10.00 \pm 0.7 \mathrm{mag}$. Throughout the paper we used the reddening law of Rieke \& Lebofsky (1985).

The reddening also can be determined from the apparent MS colors, given that the MS of young clusters is nearly vertical. Applying this method and fitting only unevolved cluster MS stars with the 4 Myr Geneva isochrone (Girardi et al. 2002) we obtained $E\left(J_{\mathrm{S}}-H\right)=1.81 \pm 0.12 \mathrm{mag}$, corresponding to $A_{K_{\mathrm{S}}}=1.19 \pm 0.12 \mathrm{mag}$ and $A_{V}=10.65 \pm 1.1 \mathrm{mag}$.

We verified these estimations once again against the spectral type classification of the WR cluster members, adopting the intrinsic WR colors of Crowther et al. (2006). The averaged color excesses for the WN stars are $E\left(H-K_{\mathrm{S}}\right)=0.56 \pm 0.07 \mathrm{mag}$ and $E\left(J_{\mathrm{S}}-K_{\mathrm{S}}\right)=1.79 \pm 0.13 \mathrm{mag}$, corresponding to $A_{K_{\mathrm{S}}}=$ $1.18 \pm 0.13 \mathrm{mag}$ and $A_{V}=10.54 \pm 1.3 \mathrm{mag}$, in reasonable agreement with our previous estimates.

Ideally, to obtain an accurate distance to the cluster we need the spectral class and respectively the absolute magnitude of some of the MS stars because they are much more uniform in comparison with the WR stars. Unfortunately, we do not have spectra of MS stars. The WN luminosities however allow us to obtain relatively good estimates of the distance. The bolometric corrections (BC) for WN stars from Crowther et al. (2006) were used to transform the luminosities to absolute $K_{\mathrm{S}}$ band magnitudes. The BCs and the distance moduli of G30 WR stars are listed in Table 3. Averaging the individual estimates, we obtain $A_{K_{\mathrm{S}}}=1.16$ and $\left(K_{\mathrm{S}}-M_{K_{\mathrm{S}}}\right)_{0}=14.31 \pm 0.35 \mathrm{mag}$, corresponding to distance of $d=7.2 \pm 0.9 \mathrm{kpc}$.

We also used the mean absolute $K_{\mathrm{S}}$-band magnitudes of the WN subtypes given in Crowther et al. (2006) to verify our distance modulus estimate. Averaging over the G30 WR members we obtained $\left(K_{\mathrm{S}}-M_{K_{\mathrm{S}}}\right)_{0}=13.58 \pm 0.35 \mathrm{mag}$. This corresponds to a shorter distance of $d=5.2 \pm 0.9 \mathrm{kpc}$.

In our further analysis we will use the larger distance modulus to the cluster because the models of WR stars yield more reliable temperature and luminosity estimates (and respectively - distance) than the spectral classification based on comparison with template spectra. Furthermore, the shorter distance modulus moves the hydrogen burning turn-on point, (where the isochrone of the PMS of $4 \mathrm{Myr}$ reaches the MS), to about 1 mag above the observed lower MS cut-off. This is in disagreement with the estimated age of the cluster (see Fig. 4 and Sect. 5.2 for details).

\subsection{Cluster age}

It is difficult to obtain a reliable age using only an isochrone MS fitting of young stellar clusters because of the nearly vertical 
linear MS locus. The first constraint comes from the lack of red supergiants in G30, evident from the CMD (Fig. 2). The stellar evolutionary models with rotation predict the onset of red supergiants at $4.5-5 \mathrm{Myr}$, defining an upper limit to the cluster age.

The WR phase is very short lived and the presence of WR stars limits the maximal age of the cluster (Meynet \& Maeder 2005). All known WR stars in G30 are of the WN6-7ha (hydrogen rich) subtype. There are indications that hydrogen rich WN7 stars are descendants of massive stars with initial masses above 50-60 $\mathcal{M}_{\odot}$ (e.g., Crowther et al. 1995). Then an upper age limit of 4-4.5 Myr can be set, independently of the exact metallicity and mass-loss scenario.

The MS turn-off MS point provides a consistency check. Our final true distance modulus $\left(K_{\mathrm{S}}-M_{K_{\mathrm{S}}}\right)_{0}=14.31 \mathrm{mag}$ suggests that the brightest unevolved star is $\mathrm{O} 9.5$ or B0 setting the cluster age to $\sim 3-4.5 \mathrm{Myr}$. This estimate is not independent, because we used WR stars to obtain the distance to the cluster.

The age of the PMS stars was determined by fitting theoretical 0.1, 1.0, 4.0, 7.0, and 10 Myr PMS isochrones from Siess et al. (2000) to the CMD (Fig. 4). Note that the PMS stars spread over a wide age range but the main locus is between the 1 and $4 \mathrm{Myr}$ isochrones. There are some stars with ages less than $1 \mathrm{Myr}$ and also a possible concentration of stars around $10 \mathrm{Myr}$ but it is too close to the depth of our photometry to draw a certain conclusion. We refrain from making more accurate conclusion about the age and the age spread because a significant fraction of the PMSs falls into the zone of photometric incompleteness. Deeper CMD is needed for that. However, we point that continuous star formation scenario or at least an extended burst cannot be excluded to have occurred in G30, based on our data.

The fraction of stars with NIR-excess correlates inversely with the stellar age, over small age ranges (Hillenbrand 2005). The vast majority $(\sim 90 \%)$ of stars older than 3-8 Myr ceases to show evidence for accretion. The fraction of IR-excess stars in a very young stellar cluster such as G30 can be used as an age indicator. We determined that for G30 the fraction is $12 \%$. The empirical calibration of Hillenbrand (2005) suggests an age of 3-4 Myr, in agreement with our previous estimations.

Similar to Hillenbrand et al. (2007) we point out that ages may be affected by the photometric uncertainties and astrophysical effects such as variability of young objects, unresolved binaries, etc. In this regard it is important to discuss the following two questions:

1) How does the distance uncertainty affect the PMS-age? - As discussed above, we have two distance modulus estimates that differ by $\approx 0.7 \mathrm{mag}$. Propagated to the PMS age this corresponds to age difference of 6-7 Myr. The shorter distance modulus gives an age of more than $10 \mathrm{Myr}$ which is inconsistent with the presence of WNha stars so the true age uncertainty is even smaller.

2) How do the photometric errors affect the age spread? The formal photometric uncertainties at the turn-on point are $\sigma\left(J_{\mathrm{S}}-K_{\mathrm{S}}\right)=0.12$ and $\sigma\left(K_{\mathrm{S}}\right)=0.10 \mathrm{mag}$. Adding the errors due to the transformations to the standard $J_{\mathrm{S}} H K_{\mathrm{S}}$ system, we see that the contribution of the photometric errors to the age range does not exceed 1-2 Myr. Therefore, most of the observed age spread is intrinsic to the PMS population.

\subsection{Initial mass function and total cluster mass}

To calculate the IMF we converted the stellar magnitudes into masses using the Geneva models. The total mass of the observed cluster members down to $\sim 2.35 \mathcal{M}_{\odot}$ (including the WR stars) is $\sim 1600 \mathcal{M}_{\odot}$ adopting a distance modulus $\left(K_{\mathrm{S}}-M_{K_{\mathrm{S}}}\right)_{0}=14.31$ and reddening $A_{K_{\mathrm{S}}}=1.16 \mathrm{mag}$. The mass estimation of $\mathrm{G} 30$ measured from the $\left(\left(J_{\mathrm{S}}-K_{\mathrm{S}}\right)_{0}, M_{K_{\mathrm{S}}}\right)$ diagram as described in Borissova et al. (2003; see their Fig. 8) gives similar result.

We also constructed a background subtracted mass function of the cluster and fitted it with a single power-law, obtaining $\Gamma=$ $-1.01 \pm 0.03$ (in these terms the Salpeter slope is $\Gamma=-1.35$ ) over the mass range $\log \mathcal{M} / \mathcal{M}_{\odot}=0.75-1.5\left(5.62-31.62 \mathcal{M}_{\odot}\right)$. The integration over this MF down to $1 \mathcal{M}_{\odot}$ combined with the masses of the WR stars leads to a total cluster mass of $\sim 3 \times$ $10^{3} \mathcal{M}_{\odot}$. Naturally, this is only a lower limit.

These results make G30 only two to three times less massive than some of the most massive young clusters in the Galaxy Arches and Quintuplet.

\subsection{Comparison with other massive clusters and position in the Galaxy}

The physical properties of the G30 together with data for Quintuplet, Arches and the Central cluster from Figer (2004) are summarized in Table 4 . Here $\mathcal{M}_{1}$ is the total cluster mass in observed stars, $\mathcal{M}_{2}$ is the total cluster mass for all stars integrated down to $1 \mathcal{M}_{\odot}$, assuming a Salpeter IMF. The radius is the radius in parsecs, from the central stellar surface density peak. The mass densities $\rho_{1}$ and $\rho_{2}$ are simply $\mathcal{M}_{1}$ and $\mathcal{M}_{2}$ divided by the cluster volume. The age and luminosity are the estimated cluster age and total luminosity.

Quintuplet shows very similar characteristics to G30. They both have a larger radius and lower stellar density than Arches and Central cluster but Quintuplet is a factor of two more massive and a factor of three more luminous than G30. The Quintuplet is also older than G30 because it contains one RSG. The properties of G30, especially the presence of WR stars, make G30 a smaller analog of Arches, Westerlund 1 and Quintuplet.

The galactocentric distance of $\mathrm{G} 30$ is $R_{\mathrm{GC}}=8.1 \mathrm{kpc}$, assuming that the Sun is at $R_{\mathrm{GC}, \odot}=8.4 \mathrm{kpc}$ from the Galactic Center (Fig. 9). G30 is located between Carina and Crux spiral arms, closer to Carina. It probably belongs to some lateral branch of the farther inner side of this arm. We see the cluster through a dust window in the nearer side of the Carina arm. Note that if the distance to the cluster is underestimated it can belong to the farther side of the main Carina arm.

\section{Summary}

We report new results of our long term project to study obscured Milky Way star clusters. We obtained deep $J_{\mathrm{S}} H K_{\mathrm{S}}$ imaging of G30, a dense stellar cluster with spectroscopically confirmed WR stars and a sizable population of young stellar objects. G30 is deeply embedded into gas and dust and suffers a reddening of $A_{V} \sim 10.5 \mathrm{mag}$. The object probably belongs to the Carina spiral arm and is located at a distance of $7.2 \pm 0.9 \mathrm{kpc}$ from the Sun. The cluster is approximately 4 Myr old. The uppermost MS stars have evolved away from the zero-age MS. G30 is massive, with a lower limit of the total mass of $3 \times 10^{3} \mathcal{M}_{\odot}$. This estimate includes only stars with masses above $\sim 1.0 \mathcal{M}_{\odot}$.

Spectral analysis and modeling of $K$ spectra for four objects show that one of these is an Ofpe/WN star, two are hydrogen rich WN6-7 stars, and the last is a WN or O-type star, all with progenitor masses above $60 \mathcal{M}_{\odot}$. The CMD suggests that there might be more WR or O type cluster members and additional observations are planned to address this possibility. G30 is a new member of the family of massive young Galactic clusters, hosting WR stars. 
Table 4. Parameters of G30 compared to the massive clusters in the Galactic Center (see Sect. 5.3 for more details). The data for Quintuplet, Arches and the Central cluster are from Figer (2004; see their Table 1). Note that the total mass and the corresponding density of G30 are only lower limits.

\begin{tabular}{lcclcccc}
\hline \hline Cluster & $\log \left(\mathcal{M}_{1}\right)$ & $\log \left(\mathcal{M}_{2}\right)$ & Radius & $\log \left(\rho_{1}\right)$ & $\log \left(\rho_{2}\right)$ & Age & $\log (\mathrm{L})$ \\
& $\mathcal{M}_{\odot}$ & $\mathcal{M}_{\odot}$ & $\mathrm{pc}$ & $\mathcal{M}_{\odot} \mathrm{pc}^{-3}$ & $\mathcal{M}_{\odot} \mathrm{pc}^{-3}$ & $\mathrm{Myr}$ & $L_{\odot}$ \\
\hline G30 & 3.2 & 3.5 & 1.36 & 2.2 & 2.5 & $3-4$ & 7.0 \\
Quintuplet & 3.0 & 3.8 & 1.0 & 2.4 & 3.2 & $3-6$ & 7.5 \\
Arches & 4.1 & 4.1 & 0.19 & 5.6 & 5.6 & $2-3$ & 8.0 \\
Center & 3.0 & 4.0 & 0.23 & 4.6 & 5.6 & $3-7$ & 7.3 \\
\hline
\end{tabular}

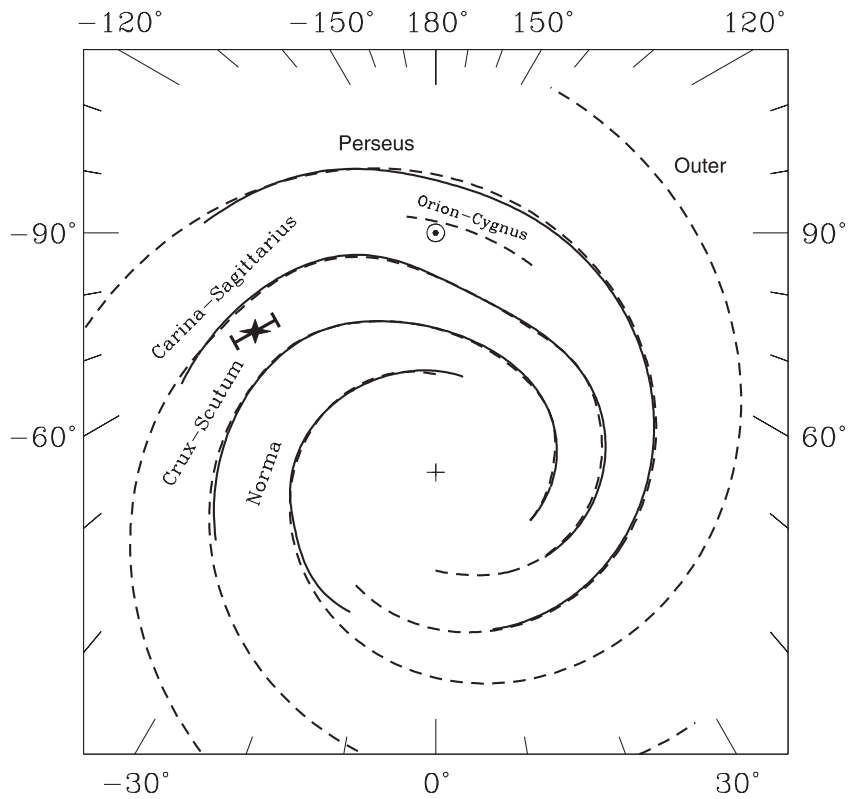

Fig. 9. Location of G30 in the Galaxy. The galactocentric distance of the cluster is $R_{\mathrm{GC}}=8.1 \pm 0.4 \mathrm{kpc}$. The error bar shows the $1 \sigma$ error of $0.9 \mathrm{kpc}$ in the distance to the Sun. The spiral structure is taken from Cordes \& Lazio (2002). The solid lines show the modified spiral model of the Galaxy from Taylor \& Cordes (1993). The dashed lines are a four-arm logarithmic spiral model combined with a local arm. The "+" sign marks the Galactic center and the Sun is labeled.

Acknowledgements. This research is partially supported by the Universidad de Valparaíso under DIPUV grant No 36/2006. The data used in this paper have been obtained with SofI/NTT at the ESO La Silla Paranal observatory. This publication makes use of data products from the Two Micron All Sky Survey, which is a joint project of the University of Massachusetts and the Infrared Processing and Analysis Center/California Institute of Technology, funded by the National Aeronautics and Space Administration and the National Science Foundation. This research has made use of the SIMBAD database, operated at CDS, Strasbourg, France. The authors would like to thank Donald Figer and Paul Crowther for placing their spectral libraries to our disposal. The authors gratefully acknowledge the very useful comments of the anonymous referee.

\section{References}

Benjamin, R., Churchwell, E., Babler, B.L., et al. 2003, PASP, 115, 953

Borissova, J., Pessev, P., Ivanov, V. D., et al. 2003, A\&A, 411, 83

Borissova, J., Ivanov, V. D., Minniti, D., et al. 2005, A\&A, 435, 95

Borissova, J., Ivanov, V. D., Minniti, D., \& Geisler, D. 2006, A\&A, 455, 923

Conti, P., Hanson, M., Morris, P., Willis, A., \& Fossey, S. 1995, ApJ, 445, 35

Cordes, J. M., \& Lazio, T. J. W. 2002 [arXiv: astro-ph/0207156]

Cotera, A., Simpson, J., Erickson, E., et al. 1999, ApJ, 510, 747

Crowther, P., \& Smith, L. 1996, A\&A, 305, 541

Crowther, P. A., Smith, L. J., Hillier, D. J., \& Schmutz, W. 1995, A\&A, 293, 427

Crowther, P., Hadfield, L., Clark, J., Negueruela, I., \& Vacca, W. 2006, MNRAS, 372,1407

Figer, D., McLean, I., \& Najaro, F. 1997, ApJ, 486, 420

Figer, D., McLean, I., \& Morris, M. 1999, ApJ, 514, 202

Figer, D. 2004, in The Formation and Evolution of Massive Young Star Clusters, ASP Conf. Ser., ed. H. J. G. L. M., Lamers, L. J., Smith, A., Nota (San Francisco: Astronomical Society of the Pacific), 322, 49

Girardi, L., Bertelli, G., Bressan, A., C., et al. 2002, A\&A, 391, 195

Hamann, W.-R., Gräfener, G., \& Liermann, A. 2006, A\&A, 457, 1015

Hanson, M. M., Conti, P. S., \& Rieke, M. J. 1996, ApJS, 107, 281

Hillenbrand, L. 2005, Review article to appear in A Decade of Discovery: Planets Around Other Stars STScI Symp. Ser. 19, ed. M. Livio [arXiv: astro-ph/0511083]

Hillenbrand, L., Bauermeister, A., \& While, R. 2007, to appear in Cool Stars 14 ed. G., van Belle, ASP Conf. Ser. [arXiv:astro-ph/0703642]

Hillier, D. J., \& Miller, D. L. 1998, ApJ, 496, 407

van der Hucht, K. A. 2001, New Astron., 45, 135

van der Hucht, K. A. 2006, A\&A, 458, 453

Ivanov, V. D., Borissova, J., Pessev, P., et al. 2002, A\&A, 349, 1

Ivanov, V. D., Kurtev, R., \& Borissova, J. 2005, A\&A, 442, 195

Koornneef, J. 1983, A\&A, 128, 84

Kudritzki, R. P., Lennon, D. J., \& Puls, J. 1995, in Science with the VLT, Proceedings of the ESO Workshop held at Garching, Germany, 28 June - 1 July 1994, ed. J. R., Walsh I. J., Danziger. (Berlin: Springer-Verlag), 246

Lamers, H. J. G. L. M., Haser, S., de Koter, A., \& Leitherer, C. 1999, ApJ, 516, 872

Maiolino, R., Rieke, G., \& Rieke, M. 1996, AJ, 111, 537

Mercer, E., Clemens, D., Meade, M., et al. 2005, ApJ, 635, 560

Meynet, G., \& Maeder, A. 2005, A\&A, 429, 581

Moorwood, A., Cuby, J. G., \& Lidman, C. 1998, The Messenger 91, 9

Rieke, G., \& Lebofsky, M. 1985, ApJ, 288, 618

Schmidt-Kaler, T. 1982, in Landolt-Borstein, New Series, Group VI, ed. K. Schaifers \& H. H.Voigt (Berlin: Springer-Verlag), 2, 1

Siess, L., Dufour, E, \& Forestini, M. 2000, A\&A, 358, 593

Skrutskie, M., Cutri, R. M., Stiening, R., et al. 2006, AJ, 131, 1163

Stetson, P. 1987, PASP, 99, 191

Taylor, J. H., Cordes, J. M., 1993, ApJ, 411, 647 